\title{
For publication
}

\section{Affinity purification of bacterial outer membrane vesicles (OMVs) utilizing a His-tag mutant}

\author{
Nathan J. Alves ${ }^{\mathrm{a}, \mathrm{b}}$, Kendrick B. Turner ${ }^{\mathrm{c}}$, Kyle A. DiVito ${ }^{\mathrm{d}}$, Michael A. Daniele ${ }^{\mathrm{e}}$, Scott A. \\ Walper $^{\mathrm{c} *}$ \\ ${ }^{a}$ National Research Council, 500 Fifth Street NW, Keck 576, Washington, DC 20001, USA \\ ${ }^{b}$ Department of Emergency Medicine, Indiana University School of Medicine, Indianapolis, IN 46202, \\ USA \\ ${ }^{c}$ Center for Bio/Molecular Science \& Engineering, Naval Research Laboratory, Washington, DC \\ 20375,USA \\ ${ }^{d}$ American Society for Engineering Education (ASEE), 1818 N Street NW, Suite 600,Washington, DC \\ 20036, USA \\ ${ }^{e}$ North Carolina State University, Joint Department of Biomedical Engineering, UNC-Chapel Hill/ NC \\ State University, 2068 Engineering Building 2,Campus Box 7911, Raleigh, NC 27695
}

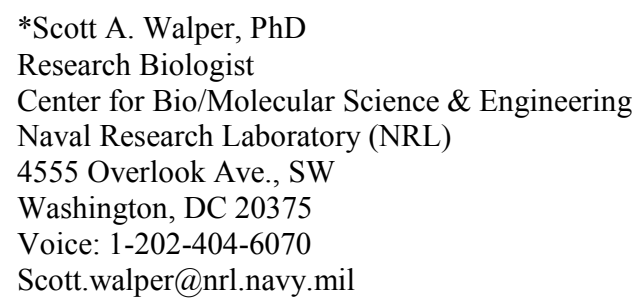

\begin{abstract}
To facilitate the rapid purification of bacterial outer membrane vesicles (OMVs), we developed two plasmid constructs that utilize a truncated, transmembrane protein to present an exterior histidine repeat sequence. We chose OmpA, a highly abundant porin protein, as the
\end{abstract}


protein scaffold and utilized the lac promoter to allow for inducible control of the epitopepresenting construct. OMVs containing mutant OmpA-His6 were purified directly from Escherichia coli culture media on an immobilized metal affinity chromatography (IMAC) NiNTA resin. This enabling technology can be combined with other molecular tools directed at OMV packaging to facilitate the separation of modified/cargo-loaded OMV from their $w t$ counterparts. In addition to numerous applications in the pharmaceutical and environmental remediation industries, this technology can be utilized to enhance basic research capabilities in the area of elucidating endogenous OMV function.

Keywords: Escherichia coli; Outer membrane vesicles (OMVs); Extracellular vesicles; Affinity purification; IMAC; His-tag.

\section{Introduction}

There is a growing list of bacterial and mammalian cell types that release extracellular vesicles (EVs), outer membrane vesicles (OMVs) or exosomes that carry out various in vivo cellular functions that include: cell-cell communication, host defense mechanisms, gene transfer and delivery of various virulence factors [1-6]. To date, all investigated Gram-positive and Gram-negative bacteria have been shown to release EV/OMV from their outer surface [7-9]. While the OMVs of Gram-negative bacteria have been extensively studied for several decades, little is known about the EVs of Gram-positive bacteria. While the proteoliposomes isolated from both Gram-positive and Gram-negative bacteria are morphologically similar, ranging in size from $30-200 \mathrm{~nm}$, the composition of these particles is highly variable, similar to the bacteria from which they are derived. Proteoliposomes are being increasingly implicated in many disease 
processes and have therefore gained much interest in the medical field for both elucidating disease progression as well as utilizing EV/OMV directly as therapeutic and vaccine pharmaceuticals [10-14]. Bacteria produce EV/OMV throughout their growth cycle, making production of these vesicles relatively simple; however, more efficient and accessible purification protocols are necessary to allow the true potential of EV/OMV and exosomes to be realized [10].

Purification of bacterial outer membrane vesicles and mammalian exosomes is a difficult process that often requires expensive equipment and complex purification protocols. Most commonly, EVs/OMVs are purified via ultracentrifugation, density gradient fractionation, immunoaffinity, precipitation or ultrafiltration techniques [15]. Ultracentrifugation, which is utilized for both pelleting EV/OMV and density gradient fractionation, requires centrifugal forces of 100,000 $\mathrm{x}$ g or greater, necessitating access to expensive and specialized centrifugation equipment. Additionally, successful pelleting of EVs/OMVs requires several hours of continuous operation at these speeds, which is a substantial time investment. Ultrafiltration techniques are less expensive, but are not as widely used in the field due to difficulties that arise when separating EVs/OMVs purely based on size [16]. The EV/OMV hydrodynamic diameter size range can sometimes significantly overlap with protein and cell fragments that they are being separated from, often resulting in poor size-based separations [8]. Immunoaffinity purification protocols utilize various immobilized EV/OMV-specific antibodies, but are limited to the repertoire of available antibodies against externally facing endogenous epitopes, that also may vary between bacterial strains and relative abundance within an EV/OMV population. Here we demonstrate the proof of concept for utilizing common immobilized metal affinity chromatography (IMAC) protein purification protocols for the affinity purification of bacterial 
EVs/OMVs through incorporation of a non-native histidine amino acid repeat sequence (Histag).

IMAC affinity purification is arguably the most commonly utilized recombinant protein purification technique for both laboratory and industrial scale applications due to its exceptionally simple and relatively inexpensive implementation into downstream protein processing [17]. The technique requires the addition of a simple multiple histidine repeat, typically 3 to 6 residues in length, to a recombinant target protein. While, typically, the His-tag is appended to the $\mathrm{N}$ - or $\mathrm{C}$-termini of the target protein, it has been shown that internal sequences can also be utilized, albeit with decreased affinity. During purification, the His-tag coordinates to an immobilized divalent metal anchored to the resin via a trinitriloacetic acid group. Unlike other affinity capture resins, the IMAC system has tunable affinity based on the species of the sequestered metal ion. Typically, IMAC resins can be functionalized with $\mathrm{Cu}^{2+}, \mathrm{Ni}^{2+}, \mathrm{Zn}^{2+}, \mathrm{Co}^{2+}$, or $\mathrm{Cd}^{2+}$ ions, each possessing their own specific affinities and benefits [18, 19].

Utilizing Escherichia coli as a model organism, we engineered two plasmid-encoded transmembrane proteins that present His-tag sequences to the outer surface of the cell membrane. We chose the porin protein OmpA as a scaffold for our construct, as the endogenous protein is highly abundant in bacterial outer membranes, and induction of the mutant OmpA constructs would not prove lethal to the bacterium [20-22]. Herein, we demonstrate that both a short 3histidine (His3) repeat within an exterior protein loop of OmpA and an extended 6-histidine (His6) repeat at the protein terminus can be used for the rapid purification of OMVs. While the bacterium chosen for these preliminary studies was a model E. coli strain, the technique described here could be broadly applied to other bacterial and mammalian species for the production and isolation of biologically derived proteoliposomes for various industrial and 
therapeutic applications following the addition of the His-tag to a membrane protein of the target organism.

\section{Materials and methods}

\subsection{Materials}

Precast Mini-PROTEAN TGX 4-15\% gradient gels were purchased from Bio-Rad (Hercules, CA, USA). A mouse anti-6xHis-tag antibody and alkaline phosphatase-conjugated anti-mouse secondary antibody were purchased from Life Technologies (Frederick, MD). GelCode blue protein stain, 1-step NBT/BCIP precipitating substrate, DiI (1,1'-dioctadecyl3,3,3',3'-tetramethylindocarbocyanine perchlorate) lipophilic membrane stain and HisPur ${ }^{\mathrm{TM}} \mathrm{Ni}^{-}$ NTA resins were purchased from Thermo Scientific (Rockford, IL, USA). All other buffer salts and reagents, including imidazole, were purchased from Sigma-Aldrich (St. Louis, MO, USA). All measurements were carried out in triplicate and data represent means \pm standard deviations.

\subsection{Construction of E. coli expression plasmids}

Genes encoding two OmpA variants containing a histidine epitope tag were synthesized by GenScript (Piscataway, NJ, USA) in a pUC57 shuttle vector with flanking NcoI and NotI restriction sites. The first construct consisted of native OmpA with the unessential C-terminal domain portion deleted and an internal His3 positioned in one of the external loops. Synthesized plasmids were digested with NcoI-HF and NotI-HF (New England Biolabs, Ipswich, MA, USA) and cloned into identical sites in the pET22b expression vector (Novagen, Billerica, MA, USA). Constructs were verified by sequencing. The Lpp' OmpA (46-159) construct originally described by Stathopoulus et al. was modified with a glycine-serine linker sequence and short multiple 
cloning site at the 3' end [23]. The gene cassette was synthesized by Genscript and cloned to a shuttle vector. The cassette was excised from the shuttle vector via XbaI - XhoI restriction digestion and cloned to the pET28 expression vector. This ligation strategy incorporated the Cterminal His6- epitope.

\subsection{OMV-conditioned media preparation}

E. coli strain BL21 (DE3) containing the pET22 OmpA-His3 or pET 28 Lpp'-OmpAHis6 plasmid was maintained on solid medium and expanded in overnight cultures in the presence of ampicillin $(100 \mu \mathrm{g} / \mathrm{mL})$ or kanamycin $(25 \mu \mathrm{g} / \mathrm{mL})$ to ensure plasmid maintenance. One $\mathrm{mL}$ of overnight culture was used to inoculate $100 \mathrm{~mL}$ of Terrific Broth in baffled culture flasks. The culture was allowed to grow for $3 \mathrm{~h}$ until an $\mathrm{OD}_{600}$ of $0.6-0.8$ was reached. Isopropyl $\beta$-D-thiogalactopyranoside was then added to a final concentration of $0.5 \mathrm{mM}$ and the culture was allowed to grow for an additional $18 \mathrm{~h}$ at $37^{\circ} \mathrm{C}$. All conditioned bacterial culture media postexpansion was centrifuged two times at 7,000 $\mathrm{xg}$ for $15 \mathrm{~min}$ at $4{ }^{\circ} \mathrm{C}$ to remove intact bacteria from the vesicle preparation. The resulting culture media was then filtered using a 0.45 $\mu \mathrm{m}$ biologically compatible filter to further remove large aggregates and undesired cellular material prior to IMAC purification.

\subsection{His-tag vesicle purification}

His-tag purification was carried out per the manufacturer's recommended protocol via a gravity-flow column or in solution batch incubation with the HisPur ${ }^{\mathrm{TM}} \mathrm{Ni}-\mathrm{NTA}$ resin. In brief, 2 $\mathrm{mL}$ of prewashed resin were added directly to prepared culture media and allowed to incubate at $4{ }^{\circ} \mathrm{C}$ for $1 \mathrm{~h}$ while mixing. The mixture was centrifuged at $700 \mathrm{x}$ g to recover the resin decanting 
the OMV-depleted culture media. The resin was then transferred to a gravity column and washed with 2-3 column volumes of PBS containing $25 \mathrm{mM}$ imidazole at $\mathrm{pH} 7.4$ collecting equivolume fractions for further analysis. Captured OMVs were then eluted from the resin with 1-2 column volumes of PBS elution buffer containing $250 \mathrm{mM}$ imidazole, being sure to collect the free solubilized OMV in equi-volume fractions.

\subsection{SDS-PAGE and western blot analysis}

The samples were run on a gradient (4-15\%) SDS-PAGE gel with a Tris-glycine running buffer under reducing conditions at $90 \mathrm{~V}$ for $90 \mathrm{~min}$, and were transferred to a nitrocellulose membrane at $90 \mathrm{~V}$ for $90 \mathrm{~min}$ in a $10 \% \mathrm{MeOH}$ transfer buffer. The membrane was probed with 1:5,000 dilution of mouse anti-6xHis-tag for $1 \mathrm{~h}$ and a 1:5,000 dilution of an alkalinephosphatase-conjugated anti-mouse secondary antibody at room temperature (RT). A chromogenic alkaline phosphatase substrate was used to detect the His-tagged mutants. SDSPAGE gels were GelCode-Blue-stained for $30 \mathrm{~min}$ and destained for $4 \mathrm{~h}$. All gel and blot images were taken on a Bio-Rad ChemiDoc imager.

\subsection{Confocal microscopy}

Vesicles were labeled with DiI (Life Technologies) following the manufacturer's protocol utilizing a 1:5,000 dilution of a $1 \mathrm{mM}$ stock solution. Samples were incubated for 20 min at $37^{\circ} \mathrm{C}$ in PBS. All transmission and fluorescence images were taken on a Nikon Eclipse TE2000-E confocal microscope using EZ-C1 spectral imaging software. All fluorescence images were taken using a 20x objective using a 650LP red filter, medium $(60 \mathrm{~m})$ pinhole and 98 
gain. Image analysis and z-stacking were performed on NIS Elements AR imaging software

3.10. All images were processed with identical parameters for direct comparison.

\subsection{SEM}

The morphologies of the vesicles were observed by scanning electron microscopy (Zeiss LEO 1550, Jena, Germany). The vesicles were snap-frozen in liquid nitrogen followed by lyophilization for $24 \mathrm{~h}$. Samples were cast onto aluminum holders and sputter-coated with ca. 5 nm of gold (Cressington 108auto, Hertfordshire, UK).

\subsection{Nanosight}

Vesicle sizing and quantitation was performed on a NanoSight LM10 system (Salisbury, UK) using NTA 2.3 Nanoparticle Tracking and Analysis software. Samples were diluted 1,000fold, and camera shutter (13.8 ms) and gain (324) were manually optimized to enhance data collection. Videos $(90 \mathrm{~s})$ were taken and frame sequences were analyzed under autoparticle detection and tracking parameters: detection threshold, pixel blur, min track length and min expected particle size. All samples were run at RT and allowed to equilibrate prior to analysis.

\section{Results and discussion}

\subsection{Design of His-tag purification constructs}

In order to utilize an affinity tag purification technique such as IMAC, a non-native histidine repeat must be incorporated into an exterior facing permissive protein domain. This protein must be expressed in relatively high abundance and also must be present in the released OMV. To demonstrate different potential ways in which IMAC OMV purification can be 
implemented, two different constructs were developed incorporating a His-tag into an exterior facing protein domain (1) mutant OmpA-His3 and (2) mutant Lpp'-OmpA-His6 (construct sequences can be found in Supporting Information Fig. S1). OmpA is a highly expressed transmembrane porin protein implicated in the transfer of small molecules across the bacterial outer membrane which is also present in subsequent OMV [21]. In the case of the OmpA-His3 mutant, a 3xHis-tag was incorporated in an exterior facing transmembrane loop to a truncated form of OmpA which lacked the periplasmic domain which binds to the peptidoglycan layer (Fig. 1A) $[22,24,25]$. This construct has been utilized by our laboratory previously in combination with a split protein system to direct the lumenal packaging of an active enzyme at either the N- or C-terminus of the recombinant truncated OmpA [26]. An internal modification that is directly linked to an external affinity tag has beneficial consequences for selectively purifying only cargo-loaded OMV in the presence of native OMV and contaminating proteins. The second construct that was developed, Lpp'-OmpA-His6, simply adds an exterior facing 6xHis-tag (Fig. 1B) to the OMV surface. In this construct, a lipoylation sequence (Lpp') directs a minimal OmpA scaffold (residues 46 - 159) which encode five transmembrane domains [2729]. Described by Stathopoulus et al., this construct allows recombinant proteins, represented in this construct as a His-tag, to be presented to the exterior surface of the bacterial outer membrane [23]. Both constructs, which are under the control of an inducible promoter, result in high abundance of the recombinant protein and His-tag in the OMV.

For all subsequent experiments involving the characterization and purification of OMV, the bacteria producing both OmpA-His mutants variants were examined. In all instances, no significant differences were observed between the two constructs. Both exhibited similar size distribution, protein content and purification efficiencies. Therefore, unless otherwise noted 
within the text, results are shown for the Lpp' OmpA His6 construct, but are representative of both mutant constructs.

\subsection{Vesicle production}

As both constructs were well tolerated by the bacterium under induction conditions, the bacterial growth and OMV production protocol were identical for both constructs. A $1 \mathrm{~mL}$ starter culture containing ampicillin or kanamycin to maintain selection was used to inoculate a $100 \mathrm{~mL}$ TB shaker flask placed at $37^{\circ} \mathrm{C}$. Once the resulting culture attained an $\mathrm{OD}_{600}$ of $0.6-0.8$ (approximately $3 \mathrm{~h}$ ), IPTG was added to the culture at a final concentration of $0.5 \mathrm{mM}$ to induce expression of the gene encoding the OmpA-His6 variant (Supporting Information Fig. S2). After an additional $18 \mathrm{~h}$ growth period, the cultures were centrifuged and passed through a $0.45 \mu \mathrm{m}$ filter to remove intact bacteria and large cellular components prior to IMAC purification. For this proof of concept in which the His-tag is associated with a protein whose production is controlled via an inducible promoter, all of the initial OMV secreted by the bacteria prior to induction will not possess a His-tag and will therefore not be captured via IMAC. There are a number of modifications to the process that can minimize the number of OMV that do not display a His-tag, such as: swapping the growth media directly preceding plasmid induction to deplete non-His-tag containing OMV, adding the inducing agent at low levels at the start of culture expansion, or by utilizing homologous recombination techniques to ensure genomic expression of the ompA-His6 at all phases of growth. The variation that is optimal would need

to be determined by the overall experimental goal, and any other limitations associated with each unique expression system. 


\subsection{OMV Purification via IMAC}

Once the intact bacteria and large bacterial fragments have been removed from the culture via centrifugation and membrane filtration, respectively, the resulting culture supernatant was subjected to IMAC purification. The culture supernatant was either applied via a gravity/pump to a prepacked IMAC resin column or, in an alternative protocol, IMAC resin was added directly to the culture supernatant in a slurry type purification protocol. Both techniques resulted in comparable capture and purification outcomes, and selection of one method over the other is largely experiment-specific. Processing of large volumes using the slurry method is often optimal, as flowing large volumes of culture supernatant over a packed resin bed can be time- and resource-intensive. After incubating the resin with the culture supernatant in a slurry type purification for $1 \mathrm{~h}$, the slurry mixture was added to a gravity flow column to capture the resin. The resin bed was then washed with fresh buffer containing $25 \mathrm{mM}$ imidazole for a total of 2-3 column volumes, being sure to collect equal volume sample fractions throughout the process. The washing step is critical to remove weakly bound protein contaminants enhancing the captured OMV purity. Captured vesicles were then eluted with 1-2 column volumes of 250 $\mathrm{mM}$ imidazole. Dialysis or membrane filtration was then used to buffer exchange the purified OMV fractions to remove the imidazole. Specifics for each method and manufacturer vary slightly, and following the supplied manufacturer's information for the slurry and packed resin processing protocols is recommended.

The chromatogram of the capture, flow-through of non-mutant OMVs/contaminants and selective release of the mutant Lpp'-OmpA-His6 OMVs by the addition of imidazole demonstrate a successful affinity separation (Fig. 2A). Vesicles were analyzed by NanoSight before and after affinity purification to verify that binding and elution from the solid resin 
surface did not negatively impact vesicle size distribution or morphology (Fig. 2B). The vesicles displayed a nearly identical size distribution pre- and post-IMAC purification. Despite the small $280 \mathrm{~nm}$ elution peak present in the native vesicle sample that did not display the Lpp'-OmpAHis6, no vesicles were captured by the Ni-NTA resin, as determined by NanoSight. It is common for there to be some off-target binding of histidine-rich proteins to the Ni-NTA column. In the presence of a high concentration of target protein/OMVs, these weakly bound off-target proteins can be largely outcompeted or removed by increasing the imidazole concentration in the wash step. Analysis of the Lpp'-OmpA-His6 OMV confirmed that the vesicles retained their native morphology, and demonstrated that a diverse OMV size distribution could be captured and eluted utilizing IMAC (Fig. 2C). Nanosight and SEM analysis of native OMVs was performed in parallel to provide for qualitative comparison of wild-type and mutant OMV (data not shown). No significant differences were observed with either method of analysis.

\subsection{Column performance}

When comparing the His-tag vesicle chromatogram with that of native vesicles, we noticed a high level of absorbance in fractions 10-25 that was not present in the native vesicle sample. This was a potential indication that weakly bound His-tag vesicles were being lost during the purification process. NanoSight analysis is limited to only determining particle size and quantity but is incapable of differentiating OmpA-His6 vesicles from native vesicles. For this reason, a western blot analysis was carried out, utilizing the included His-tag, to track whether or not all of the mutant vesicles were captured. As suggested by the His-tagged vesicle chromatogram, a significant fraction of the His-tagged vesicles were lost in the flow-through and wash fractions (Fig. 3). This was an unexpected result, as the purification was carried out well 
below the documented resin binding capacity of the commercially available Ni-NTA resin of $\leq 60 \mathrm{mg}$ of a $28 \mathrm{kDa}$ protein per $\mathrm{mL}$ of settled resin.

To investigate the apparent limited loading capacity of the Ni-NTA resin, OMVs were incubated with a lipophilic dye (DiI) and then incubated with Ni-NTA resin under OMV saturating conditions. The resin was washed to ensure that all bound OMVs were due to specific binding of the His-tag to the immobilized metal ion. The resin was then visualized on a confocal microscope and, through a combination of center slice and total intensity projections, we observed complete surface coverage of the Ni-NTA resin by the mutant vesicles, with no infiltration of the particles beyond the resin surface (Fig. 4A). There was no observed nonspecific staining, transfer of lipophilic dye to the resin beads or autofluorescence observed in unlabeled OMV or dye-exposed resin samples (Supporting Information Fig. S3). Scanning electron microscopy (SEM) was also performed on the vesicle-bound resin samples to directly visualize the vesicles on the surface (Fig. 4B).

Nearly all commercially available IMAC resins utilized for protein purification are optimized for capturing proteins that are $<300 \mathrm{kDa}$. Most proteins fall within that range; however, vesicles are considerably larger. The resin selected for this representative purification had a documented molecular weight exclusion limit of 4,000 kDa, which is typical for a $6 \%$ cross-linked agarose resin bead. To determine a theoretical molecular weight estimate for a 100 nm diameter OMV if it was a solid protein of the same size, we extrapolated using the size and weight of BSA (bovine serum albumin), which is a $66.5 \mathrm{kDa}, 14 \times 4 \times 4 \mathrm{~nm}$ protein. Using this ratio, the apparent $\mathrm{MW}$ of a $100 \mathrm{~nm}$ diameter OMV would be equivalent to $155,000 \mathrm{kDa}$, making it nearly 40 -fold larger than the MW exclusion limit of the $6 \%$ cross-linked agarose resin. With this in mind, it is not surprising that resin binding capacity is greatly reduced 
considering the OMV are too large to enter the resin pores to access internal $\mathrm{Ni}^{2+}$ ions to coordinate. While overall resin binding capacity is greatly reduced at $2 \pm 0.5 \mathrm{mg} / \mathrm{mL}$ of resin, more resin can be utilized to effectively capture more OMV, taking advantage of how easily scalable this technique is. Binding capacity of the resin may also be increased through designing an IMAC resin bead with a larger molecular weight exclusion limit specific for this application. Alternatively, materials such as Ni-NTA functionalized magnetic beads could also be employed for purification, as these materials are available in a range of sizes which could convey improved capture efficiency when combined with a batch capture/wash protocol.

\subsection{Advanced purification capabilities}

Due to the diverse roles that OMVs play in bacterial and mammalian cellular communities, there may be very discrete subpopulations within the bulk OMV population specific to performing each unique task [30]. Current OMV purification techniques largely lack the ability of differentiating subpopulations of interest from the bulk OMV populations, which is of great interest when elucidating complex cell-cell communication and disease progression pathways. Despite having reduced resin binding capacity, this technique can be implemented for purifying a well-defined OMV subpopulation from a complex mixture of non-mutant bulk OMV and free culture proteins (Fig. 5A). To demonstrate this, mutant OmpA-His6 OMVs were spiked into a culture of native OMVs and purified utilizing IMAC purification as described above. To ensure that all His-tagged OMV were captured by the resin, an excess of Ni-NTA resin was used. The OMV-bound resin was visualized by SEM to show individual OMVs bound to the resin surface (Fig. 5B). The resulting affinity separation was also analyzed by western blot, demonstrating a high capture efficiency with nearly no target OMV being lost in the flow- 
through fractions (Fig. 5C). By linking the external affinity epitope utilized for purification with internal modification sites for packaging cargo, this purification technique can also be utilized to separate cargo-loaded OMVs from non-loaded OMVs, which no other technique is currently capable of doing. This advanced purification capability has numerous applications in the production and purification of various pharmaceutical and industrial biological compounds.

In conclusion, described here is a method for the IMAC purification of bacterial outer membrane vesicles by incorporating a non-native His-tag into a permissive externally facing protein domain for capture on a Ni-NTA resin. This technique allows for selection of OMV subpopulations at the purification level, since only vesicles containing the His-tag will adhere to the resin and be purified. The technique can be further advanced through incorporation of a cleavable linker that will allow purified OMVs to be restored to their native composition for use in further experimental procedures in which the presence of a His-tag may impact results [31]. For incorporating this purification technique into a pharmaceutical downstream OMV processing scheme, it may be beneficial to incorporate the His-tag via homologous recombination to ensure that all secreted OMVs possess the affinity tag. While bacterial OMVs were utilized for the proof of concept purification described here, this technique can be adapted for use in developing analogous purification strategies for mammalian exosomes, extracellular vesicles and liposomes across a wide array of diagnostic, therapeutic, industrial and environmental remediation applications.

\section{Conflict of interest}


The authors declare no conflict of interest.

\section{Acknowledgments}

This research was funded by the Office of Naval Research through Core funds provided to the Naval Research Laboratory.

Fig. 1. A) Schematic representation of the bacterial OMVs displaying the truncated OmpA embedded in the lipid bilayer displaying the location of the externally facing His-tag and internally modifiable N- and C-termini. B) Schematic representation of Lpp'-OmpA-His6 construct. C) Representation of a complex OMV containing numerous surface modifications, transmembrane proteins and loaded cargo.

Fig. 2. A) Chromatogram for a representative purification of His-tag vesicles purified directly from $E$. coli culture media via IMAC Ni-NTA resin showing the capture (Fractions 1-10), flowthrough of non-mutant OMV and contaminants (Fractions 11-44) and selective release of the Lpp'-OmpA-His6 OMV by addition of imidazole (Fractions 45-55). B) NanoSight histograms of pre- and post-IMAC purified vesicles demonstrating no apparent impact to vesicle size post binding and elution from the Ni-NTA resin. No native OMVs were observed in the elution 
fraction from native OMV Ni-NTA purification. C) SEM image of purified Lpp'-OmpA-His6 OMV.

Fig. 3. Western blot of the elution profile of Lpp'-OmpA-His6 mutant vesicles from Ni-NTA resin utilizing an anti-His-tag antibody. This separation demonstrates oversaturation of the column in which much of the mutant vesicles are lost in the initial flow-through. Captured vesicles were washed and subsequently eluted with the addition of imidazole. Despite exceeding the column capacity, the separation of flow-through and elution by a wash region containing very few mutant OMV demonstrates successful capture and elution of target OMV.

Fig. 4. A) Confocal images of the transmission (left) and center slice fluorescence (right) of DiI dye labeled His-tagged OMV showing capture by the Ni-NTA resin. These saturated resin beads are representative of purification carried out in Fig. 3 in which a large portion of mutant OMVs were lost in flow-through. This demonstrates how OMVs are too large to permeate into the resin which reduces the overall resin binding capacity. B) SEM image of the OMV-saturated NiNTA resin surface showing complete blocking of the resin pores. 
Fig. 5. A) Schematic representation of selective affinity purification of His-tag protein-loaded OMVs from native non-loaded OMV and other culture media contaminants. B) SEM image of high Ni-NTA resin to OmpA-His3 vesicle ratio. This technique allows direct visualization of the OmpA-His3 vesicles bound to the surface of the Ni-NTA resin. C) Western blot of the elution profile of OmpA-His3 mutant vesicles from Ni-NTA resin utilizing an anti-His-tag antibody. This separation demonstrates a high capture efficiency and recovery of a minutely expressed mutant His-tagged vesicle from a complex mixture such as conditioned bacterial culture media.

\section{References}

[1] Berleman J, Auer M. The role of bacterial outer membrane vesicles for intra- and interspecies delivery. Environmental Microbiology 2013;15:347-54.

[2] Eddy JL, Gielda LM, Caulfield AJ, Rangel SM, Lathem WW. Production of outer membrane vesicles by the plague pathogen Yersinia pestis. PLoS One 2014;9:e107002.

[3] Ellis TN, Kuehn MJ. Virulence and immunomodulatory roles of bacterial outer membrane vesicles. Microbiol Mol Biol Rev 2010;74:81-94.

[4] Kulkarni HM, Jagannadham MV. Biogenesis and multifaceted roles of outer membrane vesicles from Gram-negative bacteria. Microbiology 2014;160:2109-21.

[5] Thery C, Zitvogel L, Amigorena S. Exosomes: Composition, biogenesis and function. Nature Reviews Immunology 2002;2:569-79.

[6] Thery C, Ostrowski M, Segura E. Membrane vesicles as conveyors of immune responses. Nature Reviews Immunology 2009;9:581-93. 
[7] Avila-Calderon ED, Araiza-Villanueva MG, Cancino-Diaz JC, Lopez-Villegas EO, Sriranganathan N, Boyle SM, et al. Roles of bacterial membrane vesicles. Arch Microbiol 2015;197:1-10.

[8] Kulp A, Kuehn MJ. Biological functions and biogenesis of secreted bacterial outer membrane vesicles. Annu Rev Microbiol 2010;64:163-84.

[9] Beveridge T. Structures of Gram negative cell walls and their derived membrane vesicles. J Bacteriol 1999;181:4725-33.

[10] Alves NJ, Turner KB, Medintz IL, Walper SA. Emerging therapeutic delivery capabilities and challenges utilizing enzyme/protein packaged bacterial vesicles. Therapeutic delivery 2015;6:873-87.

[11] Chen DJ, Osterrieder N, Metzger SM, Buckles E, Doody AM, DeLisa MP, et al. Delivery of foreign antigens by engineered outer membrane vesicle vaccines. Proc Natl Acad Sci U S A 2010;107:3099-104.

[12] Gujrati V, Kim S, Kim S-H, Min JJ, Choy HE, Kim SC, et al. Bioengineered bacterial outer membrane vesicles as cell-specific drug-delivery vehicles for cancer therapy. ACS Nano 2014;8:1525-37.

[13] Collins BS. Gram-negative outer membrane vesicles in vaccine development. Discovery Medicine 2011;12:7-15.

[14] Acevedo R, Fernandez S, Zayas C, Acosta A, Elena Sarmiento M, Ferro VA, et al. Bacterial outer membrane vesicles and vaccine applications. Frontiers in Immunology 2014;5.

[15] Klimentova J, Stulik J. Methods of isolation and purification of outer membrane vesicles from gram-negative bacteria. Microbiol Res 2015;170C:1-9. 
[16] Alves NJ, Cusick W, Stefanick JF, Ashley JD, Handlogten MW, Bilgicer B. Functionalized liposome purification via Liposome Extruder Purification (LEP). Analyst 2013;138:4746-51. [17] Cheung RCF, Wong JH, Ng TB. Immobilized metal ion affinity chromatography: a review on its applications. Applied Microbiology and Biotechnology 2012;96:1411-20.

[18] Porath J. Immobilized metal-ion affinity-chromatography. Protein Expr Purif 1992;3:26381.

[19] Kuo WHK, Chase HA. Exploiting the interactions between poly-histidine fusion tags and immobilized metal ions. Biotechnology Letters 2011;33:1075-84.

[20] Chatterjee SN, Chaudhuri K, Gram-negative bacteria: the cell membranes, in Outer Membrane Vesicles of Bacteria, Springer, Berlin, 2012, pp 15-34.

[21] Wang Y. The function of OmpA in Escherichia coli. Biochem Biophys Res Commun $2002 ; 292: 396-401$.

[22] Danoff EJ, Fleming KG. The soluble, periplasmic domain of OmpA folds as an independent unit and displays chaperone activity by reducing the self-association propensity of the unfolded OmpA transmembrane beta-barrel. Biophys Chem 2011;159:194-204.

[23] Stathopoulos C, Georgiou G, Earhart CF. Characterization of Escherichia coli expressing an Lpp'OmpA(46-159)-PhoA fusion protein localized in the outer membrane. Applied Microbiology and Biotechnology 1996;45:112-9.

[24] Kleinschmidt JH, den Blaauwen T, Driessen AJM, Tamm LK. Outer membrane protein A of Escherichia coli inserts and folds into lipid bilayers by a concerted mechanism. Biochemistry 1999;38:5006-16.

[25] Alves NJ, Turner KB, Medintz IL, Walper SA. Protecting enzymatic function through directed packaging into bacterial outer membrane vesicles. Scientific Reports 2016;6:24866. 
[26] Alves NJ, Turner KB, Daniele MA, Oh E, Medintz IL, Walper SA. Bacterial nanobioreactors-directing enzyme packaging into bacterial outer membrane vesicles. ACS Applied Materials \& Interfaces 2015;7:24963-72.

[27] Ghrayeb J, Inouye M. 9 Amino-acid-residues at the NH2-terminal of lipoprotein are sufficient for its modification, processing and localization in the outer-membrane of Escherichiacoli. J Biol Chem 1984;259:463-7.

[28] Inouye S, Wang S, Sekizawa J, Halegoua S, Inouye M. Amino-acid sequence for peptide extension on pro-lipoprotein of Escherichia-coli outer membrane. Proc Natl Acad Sci U S A 1977;74:1004-8.

[29] Verhoeven GS, Alexeeva S, Dogterom M, den Blaauwen T. Differential bacterial surface display of peptides by the transmembrane domain of OmpA. Plos One 2009;4:e6739.

[30] Wieser A, Storz E, Liegl G, Peter A, Pritsch M, Shock J, et al. Efficient quantification and characterization of bacterial outer membrane derived nano-particles with flow cytometric analysis. Int J Med Microbiol 2014;304:1032-7.

[31] Li YF. Self-cleaving fusion tags for recombinant protein production. Biotechnology Letters 2011;33:869-81. 
A

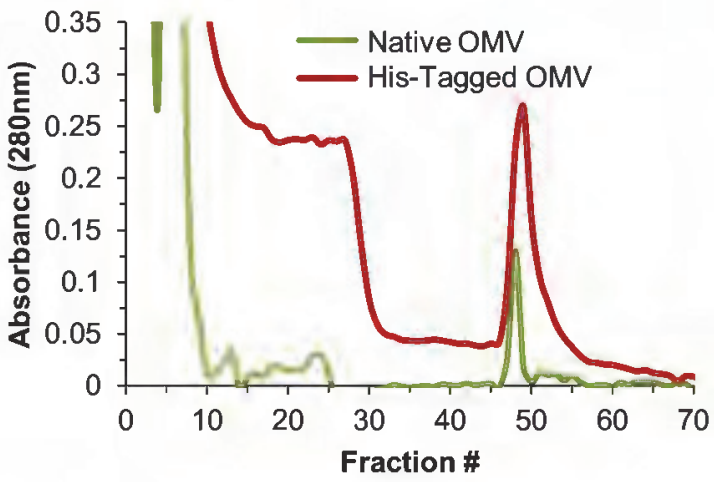

B

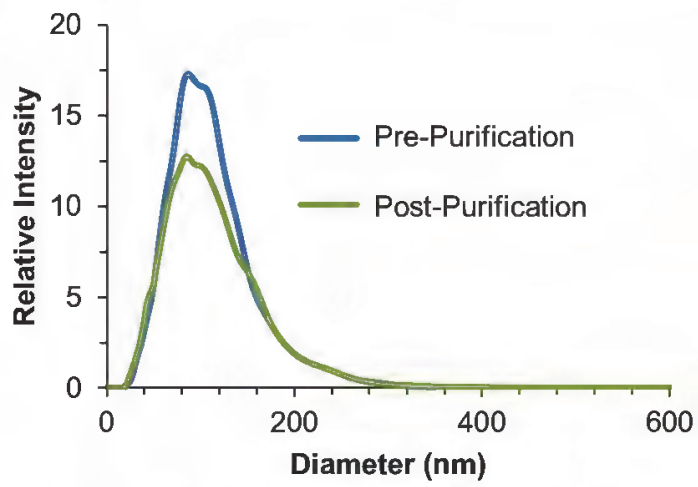

C

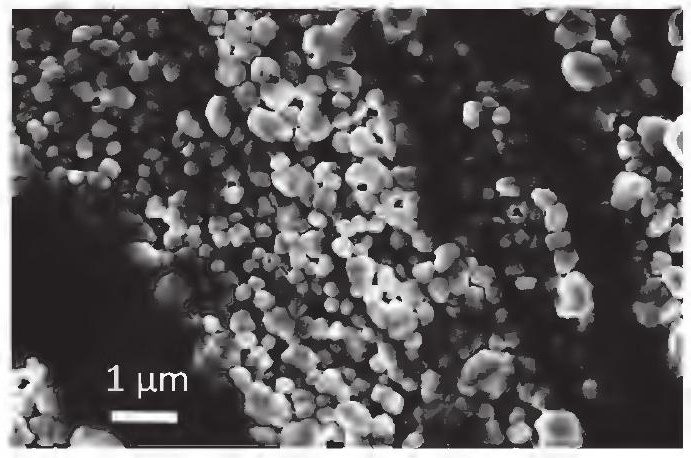




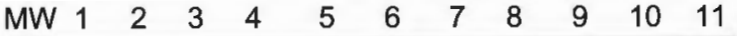

100

75

50

37

25

20

15

10

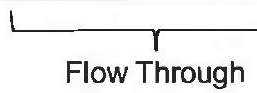

$儿$

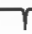

Wash

Elution 


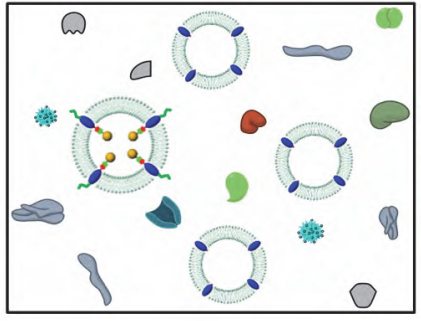

Mixed OMV + Contaminants

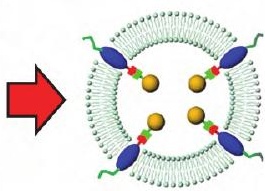

Purified His-Tag OMV

B

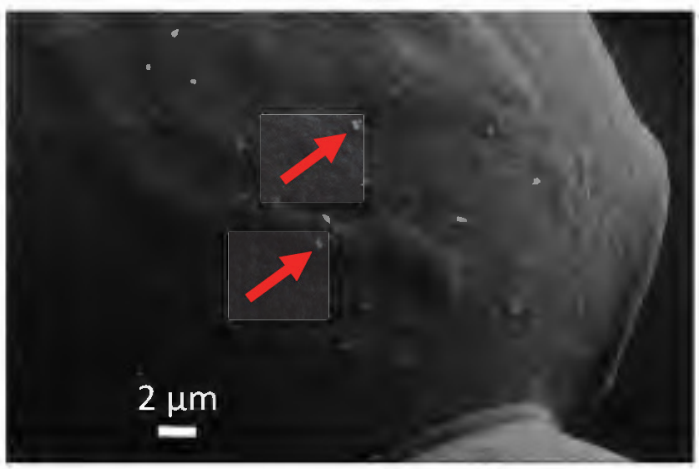

$\begin{array}{llllllllllll}\text { MW } & 1 & 2 & 3 & 4 & 5 & 6 & 7 & 8 & 9 & 10 & 11\end{array}$

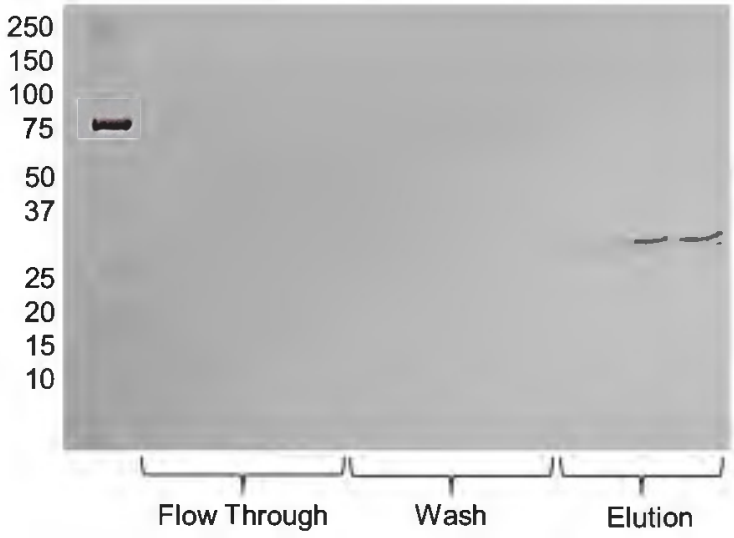

